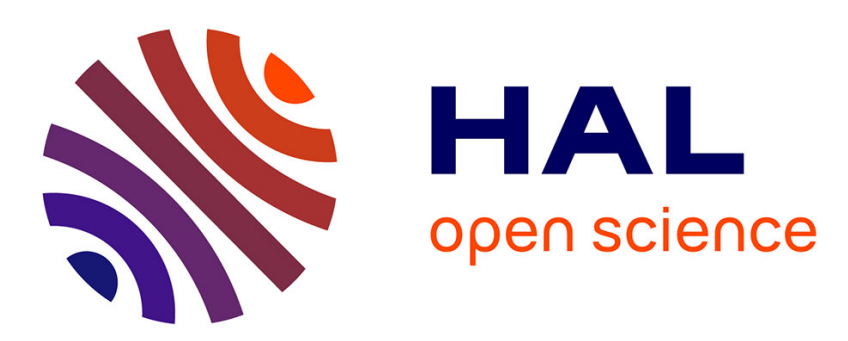

\title{
Serverless lightweight mutual authentication protocol for small mobile computing devices
}

Collins Isdory Mtita, Maryline Laurent, Pascal Daragon

\section{To cite this version:}

Collins Isdory Mtita, Maryline Laurent, Pascal Daragon. Serverless lightweight mutual authentication protocol for small mobile computing devices. NTMS 2015: 7th International Conference on New Technologies, Mobility and Security, Jul 2015, Paris, France. pp.1 - 5, 10.1109/NTMS.2015.7266521. hal-01285358

\section{HAL Id: hal-01285358 \\ https://hal.science/hal-01285358}

Submitted on 9 Mar 2016

HAL is a multi-disciplinary open access archive for the deposit and dissemination of scientific research documents, whether they are published or not. The documents may come from teaching and research institutions in France or abroad, or from public or private research centers.
L'archive ouverte pluridisciplinaire HAL, est destinée au dépôt et à la diffusion de documents scientifiques de niveau recherche, publiés ou non, émanant des établissements d'enseignement et de recherche français ou étrangers, des laboratoires publics ou privés. 


\title{
Serverless Lightweight Mutual Authentication Protocol for small mobile Computing Devices
}

\author{
Collins MTITA ${ }^{1,2}$, Maryline LAURENT², Pascal DARAGON ${ }^{1}$ \\ ${ }^{1}$ TRAXENS S.A.S, Marseille, France \\ ${ }_{1}$ \{c.mtita, p.daragon\}@traxens.com \\ ${ }^{2}$ Institut Mines-Télécom/Télécom SudParis, CNRS UMR 5157 SAMOVAR, Évry, France \\ ${ }^{2}$ \{collins.mtita, maryline.laurent\}@telecom-sudparis.eu
}

\begin{abstract}
Small and inexpensive computing devices are becoming potential players in the Internet arena. Smart constrained devices are used for collecting or generating information which is later relayed to the designated servers. The information gathered must be adequately protected against all kinds of attacks during storage and transmission. However, most smart constrained devices have limited resources and unable to run most of the classical protocols that provide robust security.

This article presents a lightweight mutual authentication protocol for resource constrained heterogeneous computing devices with high mobility. Our solution is novel as it facilitates two resource constrained devices to autonomously perform mutual authentication and establish a session key without sharing common parameters beforehand. Our proposed lightweight protocol is symmetric and uses simple primitives such as XoR, comparison and keyed-Hashed Message Authentication Code (HMAC) during mutual authentication. Moreover, our protocol requires minimum storage for storing few keys and parameters locally. The protocol is formally verified using AVISPA tool.

Keywords: Security, Low energy footprint, lightweight security, Validation, Mutual Authentication, Heterogeneous Constrained Computing Devices, Symmetric Protocol
\end{abstract}

\section{Introduction}

S mart constrained computing devices are slowly becoming major players in the Internet arena dominated by the era of ubiquitous computing. There is a steady emergence of platforms [5], [18 that allow smart constrained computing devices to independently interconnect and interact with other parties over the Internet in ways that were not initially envisaged.

Before harnessing the full potential brought by these technologies into our daily lives we must first address core security and privacy issues pertaining to the information stored and exchanged. Technology growth forces smart constrained devices to release the information gathered on real-time basis. However, this poses new challenges [26] [7], [31] because constrained devices do not have enough capabilities to provide access rights to various external entities [3] without the help of a powerful central server.

Using the current centralized server models, services provided by smart devices in remote geographical locations may be regularly interrupted due to the lack of reliable connectivity between smart computing devices and centralized server. Likewise, it is challenging to grant access control when the interaction between smart constrained devices and external parties cannot be known in advance, nonetheless, appropriate access rights must be assigned to each requesting party in order to allow smooth authentication and reliable access to the information. In such cases, a reliable solution necessitates authentication to proceed without a persistent connection to the central server.

In this paper we address the issue of securing data stored or exchanged by smart computing devices in the absence of the centralized server. We propose a serverless mutual authentication protocol for mobile resource-constrained devices. Our protocol is symmetric lightweight and uses simple primitives like keyed-Hash Message Authentication Code (HMAC), XoR and comparison operations to validate authenticity of all parties accessing data in smart devices.

The rest of this paper is organized as follows, section 2 presents a scenario pertaining to the protocol discussed in this article while section 3 presents related work. Section 4 discusses our proposed protocol and section 5 validates our protocol using AVISPA tool Protocol's analysis is done in section 6 and section 7 concludes.

\section{Serverless Protocol Scenario and Features}

Imagine a scenario where tracking devices are attached to containers in transit from one geographic location to another. Tracking devices collect and log crucial information in the course of the trip but also serve as identifiers for respective containers they are attached to. On transiting custom checkpoints, controllers possessing mobile personal digital assistants (PDAs) must authenticate to tracking devices before identifying and accessing stored information, for instance container number, type of merchandise, destination e.t.c. But, PDAs must obtain prior authorization to access information in tracking devices from a remote central server, which is only accessible via the network.

One way to accomplish this task requires PDAs to establish persistent connections to the central server during the authentication process. However, in case of network unavailability, the protocol breaks down and PDAs cannot communicate with tracking devices. The journey must be delayed until connection is established. This leads to unnecessary inconveniences.

Alternatively, PDAs may connect once or twice to the central server, whenever the network is available during the day, download appropriate authentication data and store them locally. In that case, PDAs can autonomously authenticate tracking devices without the need of a persistent connection to the central server. This guarantees reliable authentication between legitimate PDAs and tracking devices, even when the central server is unavailable or incapacitated.

Our proposed scenario's network architecture contains three types of communicating parties - Central Server (CS), Lightweight Responder $(\boldsymbol{L R})$ and Lightweight Initiator $(\boldsymbol{L I})$.

1. Central Server $(C S)$ : A powerful server with unlimited resources that controls access between $L I$ and $L R$ s and administers $L R$ s.

2. Lightweight Initiator (LI): A terminal accessing information stored in LRs. LI has enough resources in terms of computing power and storage space.

3. Lightweight Responder $(L R)$ : A resource constrained terminal with limited resources in terms of storage capacity, computation power and energy. $L R$ constantly collects and stores sensitive data. Each $L R$ has a secret key $K_{C}$ provided by $C S$ and a static timestamp $T_{C}$ initialized by $C S$ during device setup. LRs can be RFID Tags, NFC Tags or other constrained data capturing devices. $L R$ is the most important player in the scenario that our proposed protocol aims at protecting.

In our scenario, LRs are geographically distributed in form of clusters. A cluster is a collection of $L R$ s within a small geographic region sharing the same secret key $K_{C}$ that is used to verify authenticity of external entities interacting with $L R$ s within a cluster. $L I$ is an external device accessing information stored in $L R s$ within a given cluster. Prior to accessing LRs in a cluster, LI must securely connect to $C S$ and request authorization to access $L R s$ within a given 
cluster. $L I^{\prime}$ 's request contains its geographic position, authenticating parameters and the identifier $I D_{L}$. As $C S$ knows all legitimate $L R s$ and $L R$ clusters, their secret keys and respective geographic positions, it replies to $L I$ by providing necessary parameters for accessing requested cluster of $L R$ s. Some of these parameters are $L I$ 's key $K_{L}$, access rights $A R$, Time Window $W_{S}$ and list of temporary identities $L_{j}$ for $L R s$ within a cluster.

Our proposed scenario has the following basic features:

1. Constrained resources: LRs cannot memorize credentials and access control rights [10], [36] for each $L I$ due to limited resources. 2. No prior knowledge of each other: $L I$ and $L R$ have no knowledge of each other's existence prior to mutual authentication phase.

3. Absence of Server during authentication session: CS may not be available during mutual authentication phase due to unreliable connectivity.

4. Scalability: Within a cluster, LIs and LRs can freely interact.

5. Trust relationship: $L R$ and $L I$ do not have mutual trust. The trust relationship is built during authentication with the help of $C S$, even if it is not actively involved in the authentication process.

\section{Related Work}

Various security protocols for constrained devices have been put forward, most of which advocate the availability of a persistent connection to the central server during authentication [1], [24]. However, connection-oriented model faces major limitations [32], especially in the era of ubiquitous computing, where devices can be located anywhere without a guarantee of reliable connectivity [20]. Intermittent connectivity may render protocols unavailable during long periods of time. This is one of the major reasons behind the efforts to seek alternative and reliable solutions. This section analyzes some of the proposed serverless protocols for constrained devices.

In 2008, Tan et al. [32] first introduced Serverless search and authentication RFID protocol. Tan et al. suggested that protocol's reliability can be guaranteed by eliminating a persistent link between an RFID reader and the backend during authentication process. Their proposal included downloading necessary information that can allow a reader to autonomously authenticate tags. However, Tan et al.'s protocol was found to be vulnerable to traceability, impersonation and privacy attacks by the authors of [30]. In 2009 Lin et al. |21] proposed a serverless RFID authentication protocol which improved the computational performance of Tan et al. protocol. However, as pointed out by authors of [19], Lin et al.'s, like with Tan et al.'s, proposed protocol performed a one sided authentication, where the reader authenticates the tag but the tag does not authenticate the reader. Moreover, authors of [19] reveal that Lin et al.'s protocol is also vulnerable to impersonation attack.

Hoque et al. [15] proposed a serverless, untraceable authentication, and forward secure protocol for RFID tags. Hoque et al claim that their protocol safeguards both reader and tags against common attacks without the need of backend server's intervention. But, Deng et al. [11] found that Hoque et al.'s authentication protocol was susceptible to data desynchronization attack and proposed an improvement. Deng et al.'s proposed authentication protocol was designed to withstand data desynchronization attacks, but the authors of [29] found that Deng et al.'s protocol is still vulnerable to data desynchronization attack after two protocol runs.

The authors of [2] proposed ERAP, the ECC based RFID Authentication Protocol, which performs mutual authentication between the reader and the authorized RFID tags without the need of persistent connection to the backend server. This scheme was found vulnerable to denial of service attack by authors of [23]. The authors of [33 propose (HOA) HLR Offline Authentication, the authentication scheme suitable for low-power mobile devices based on ECC. However this protocol requires prior knowledge of each communicating entity and too much CPU and memory resources as tags must perform ECC point multiplication and modular operations.

The idea to use timestamp during authentication of constrained devices was first introduced by Tsudik [34]. Tsudik's idea was quite novel but curious due to the fact that most of the constrained devices do not have embedded clocks to keep track of the time. Tsudik suggested that RFID reader should periodically broadcast timestamp of its current time. A tag, within the proximity of reader, receives and compares the broadcast timestamp with a stored timestamp value. If the former is strictly greater than the latter, the tag computes a response derived from its permanent key and the new timestamp. Otherwise, the tag replies with a pseudorandom to confuse the adversary and thwart narrowing attacks. According to Tsudik [34], a narrowing attack occurs when the adversary queries a tag with a particular timestamp and then later tries to identify the same tag by querying a candidate tag with a timestamp slightly above the previous one.

However, Tsudik's idea is vulnerable to Denial of Service (DoS) attacks. An adversary can easily desynchronize the tag by sending the timestamp value that is ahead of time. This idea was later improved by authors of [8] by moving the attack from the resource constrained tag to the powerful backend server. The improvement aimed at thwarting DoS attacks against the tags but it also resulted to an exhaustive search to the backend server.

\section{Serverless Mutual Authentication Protocol}

Our protocol leverages on the power of $C S^{\prime}$ s knowledge on $L R$ clusters with their respective credentials to facilitate authentication, even though CS does not actively participate during mutual authentication phase. To further facilitate authentication between two constrained devices, our protocol uses timestamp as one of the parameters during authentication between $L I$ and $L R$.

\subsection{Security and Privacy Requirements}

Our proposed authentication protocol must fulfill the following requirements.

1. Mutual Authentication: Our protocol must perform mutual authentication between $L R$ and $L I$ prior to data exchange session in order to thwart impersonation attacks.

2. Key Exchange: Our protocol must securely establish a common key between $L R$ and $L I$ to be used during data exchange session.

3. Freshness: Messages exchanged during mutual authentication session must be fresh. Our protocol uses timestamp and random values to enforce freshness.

\subsection{Privacy and Security threat models}

Several attacks may be launched against our protocol. We propose privacy and security games to model possible threats and demonstrate how relisient our protocol can be against attacks.

Game 1: $\beta$ masquerades as LI

- Phase 1.1: $\beta$ eavesdrops several exchanges between one or more $L R$ and various $L I$ s.

- Phase 1.2: $\beta$ sends message $b_{1}$ and then message $b_{3}$ to $L R$. $\beta$ wins the game if he can reply $L R$ with a valid message $b_{3}$. Game 2: $\beta$ tracks $L R_{i}$

- Phase 2.1: $\beta$ colludes with a legitimate device $L I$ and listens to exchanges between $L I$ and responder $L R_{1}$ and then between $L I$ and $L R_{2}$.

- Phase 2.2: Challenger selects $L R_{i}, i \in\{1,2\}, \beta$ listens to exchanges between $L I$ and $L R_{i}$, and $\beta$ sends a guess $i$ value to the challenger.

$\beta$ wins the game if $i$ is correct. The protocol is considered private if $\beta$ cannot win the game with probability greater than 0.5 .

Game 3: $\dot{\beta}$ depletes $L R$ 's resources

- Phase 3.1: $\beta$ eavesdrops messages $b_{1}$ between LIs and LRs.

- Phase 3.2: $\beta$ sends forged $b_{1}$ messages to a targeted $L R$ within a cluster.

$\beta$ wins the game if he can successfully deplete $L R$ 's battery within 12 hours (This corresponds to an overnight attack). 


\subsection{Assumptions}

1. All LRs running our protocol are capable of performing simple primitives such as Keyed-Hash Message Authentication Code (HMAC), comparison and XOR. In this article, HMAC is based on SHA1 (Secure Hash Algorithm 1) [13] and its output is truncated to the 128 bits (energy saving). However, our protocol can work with any HMAC. 2. Pseudo Random Number Generator (PRNG) and HMAC are robust. 3. CS and LI share secret parameters used to launch a secure channel, e.g. via secure protocol https, for exchanging secret information.

4. $C S$ shares a secret key $K_{C}$ with each $L R$. $K_{C}$ is common among all legitimate $L R$ s within a specific cluster.

5. $L I$ 's periodic key $K_{L}$ is used to solicit $L R$ s within a specific cluster sharing the same key $K_{C}$, provided Time Window $W_{S}$ is still valid.

\subsection{Protocol Notations}

Table 1 presents notations used in the protocol. Access Rights (AR) is a code for access levels and rights that $L I$ has pertaining to the data stored in $L R$. In our protocol, $A R$ is represented in form of a code, like Unix file permissions, with Read, Write and Execute options. $H_{K}$ is a secret parameter that $L I$ uses to securely pass initial parameters to $L R$ s. $L R^{\prime}$ s static timestamp $T_{C}$ is initialized by a default timestamp value $T_{\text {init }}$ during initial configuration by $C S$. Time Window $W_{S}=\left[T_{0} \| T_{Z}\right]$ is a 64 bits parameter made from two timestamps, 32 bits start timestamp $T_{0}$ and 32 bits end timestamp $T_{Z}$ parameters used to show $K_{L}$ 's validity.

Table 1: Protocol notations with size estimations

\begin{tabular}{llr}
\hline Parameter name & Symbol & Bits \\
\hline LI's system Time & $T_{L I}$ & 32 \\
LR's stored timestamp & $T_{C}$ & 32 \\
Start Time Window & $T_{0}$ & 32 \\
End Time Window & $T_{Z}$ & 32 \\
Time Window & $W_{S}$ & 64 \\
LI's Identifier & $I D_{L}$ & 128 \\
LI's Key & $K_{L}$ & 128 \\
LR's identifier & $I d_{i}$ & 128 \\
LR's cluster Key & $K_{C}$ & 128 \\
Derived session key & $K_{S}$ & 128 \\
Timestamp signature & $H_{T}$ & 128 \\
Random Value & $R_{1}$ & 128 \\
Access Rights & $A R$ & 128 \\
LI's secret code & $H_{K}$ & 128 \\
List of $L R$ s temporary identities & $L_{j}$ & - \\
\hline
\end{tabular}

\subsection{Protocol Description}

The proposed protocol operates in two phases. Phase A involves interaction between $L I$ and $C S$, and Phase $B$ involves interaction between $L I$ and $L R$.

\section{Phase A: Interaction between CS and LI}

$L I$ requests authorization from CS to access information stored in $L R$ s. CS also uses this phase to synchronize time with $L I$. $L I$ securely connects to $C S$ and sends message $a_{1}$ for requesting authorization to access a cluster of $L R s$ in its vicinity. Message $a_{1}$ contains $L R^{\prime} \mathrm{s}$ identifier $I D_{L}$ and its geographic location. $C S$ receives $L I^{\prime}$ s request and generates a list $L_{j}$ of temporary identities for each $L R$ within a cluster (cf. Line (4)), $L I$ 's key $K_{L}$ (cf. Line (5)) and secret code $H_{K}$ (cf. Line (6)). CS sends back message $a_{2}$ via the established secure channel (cf. Line (7)). LI receives and decrypts message $a_{2}$ from CS containing $K_{L}, H_{K}, A R, W_{S}$ and $L_{j}$.

$$
\begin{aligned}
& L I \stackrel{a_{1}}{\longrightarrow} C S \quad: \text { Request } \\
& \text { CS : Get Time Window: } W_{S}=\left[T_{0}, T_{Z}\right] \\
& \forall \text { LI generate } I d_{T e m p}=H M A C_{I d_{i}}\left(T_{0}\right) \\
& \text { Create list } L_{j}=\left\{I d_{T e m p_{1}}, I d_{T e m p_{2}}, \ldots, I d_{T e m p_{i}}\right\} \\
& K_{L}=H M A C_{K_{C}}\left(I D_{L}\|A R\| W_{S}\right) \\
& H_{K}=H M A C_{K_{C}}\left(W_{S}\right)
\end{aligned}
$$

$$
\begin{array}{rll}
L I \stackrel{a_{2}}{\longleftarrow} C S & : & K_{L}, H_{K}, W_{S}, A R, L_{j} \\
L I & : & \text { Get } K_{L}, H_{K}, W_{S}, A R, L_{j}
\end{array}
$$

Listing 1: Authorization phase between CS and LI

\section{Phase B: Mutual Authentication Between LR and LI}

Mutual authentication between $L I$ and $L R$ is completed in three exchanges without assistance from $C S$.

$L I$ encrypts its identifier $I D_{L}$ using its secret code $H_{K}$ (cf. Line (10)) and calculates timestamp signature $H_{T}$ (cf. Line (11)). LI broadcasts message $b_{1}$ containing $e_{I D}$, timestamp $T_{L I}$, timestamp signature $H_{T}$, together with Access Rights AR, and Time Window $W_{S}$ from CS. $L I$ precalculates and stores in a table the values of $H_{1_{i}}$ corresponding to each $I d_{T e m p_{i}}$ (cf. Line (12)). This step facilitates the search of $H_{1_{i}}$ values once message $b_{2}$ is received.

Upon receipt of message $b_{1}, L R$ verifies $T_{Z}$ and $T_{L I}$ against its stored timestamp $T_{C}$, where $T_{Z}$ is last 32 bits of timestamp $W_{S}$ as explained in Section $4.4 \quad L I$ aborts the current session if the timestamp parameters are not correct.

\section{B1. Validation of Key $K_{L}$}

Each valid $L R$ can easily calculate $H_{K}$ using $W_{S}$ and $K_{C}$ (cf. Line (16)), then use it to recover $I D_{L}^{\prime}$ (cf. Line (17)) which is used to calculate $K_{L}^{\prime}$ (cf. Line (18)). Using $K_{L}^{\prime}, L R$ authenticates timestamp signature $H_{T}$ (cf. Line (20)). If $H_{T}$ is invalid, the session is terminated, else $L R$ updates its timestamp $T_{C}$ (cf. Line (21)). A valid $H_{T}$ in Line (20) proves the validity of key $K_{L}$ used by $L I$.

After verification of $K_{L}, L R$ generates a temporary identity using its identifier $I d$ and $T_{0}$ from $W_{S}$ (cf. Line (24)) and also generates a signature $H_{1}$ for $T_{L I}$ (cf. Line (25)). Likewise, $L R$ generates a random $R_{1}$ (cf. Line (26)), which is used to calculate a cipher $e_{1}$ and a signature $H_{2}$ (cf. Lines (27) and (28)). Cipher $e_{2}$ and signatures $H_{1}$ and $\mathrm{H}_{2}$ are sent to $L I$ via message $b_{2}$.

\section{B2. Authentication of $L R$}

$L I$ receives message $b_{2}$ and quickly finds a value that corresponds to a signature $H_{1}$ within a table of precaculated $H_{1_{i}}$ values. Once found, $R_{1}^{\prime}$ can be easily recovered as indicated in Line (31) and its validity tested by comparing calculated $H_{2}^{\prime}$ against the received $H_{2}$. Validity of Line (33) authenticates $L R$ in $L I$ and prompts $L I$ to calculate $H_{R}=H M A C_{I d_{\text {Temp }}}\left(R_{1}^{\prime}\right) . H_{R}$ is sent to $L R$.

\section{B3. Authentication of $L I$}

$L R$ receives and verifies $H_{R}$, if correct, $L R$ authenticates $L I$ (cf. Line (40)).

\section{B4. Generation of session key $K_{S}$}

Lines (37) and (41) show generations of key $K_{S}$ on $L I$ and $L R$ respectively after a successful mutual authentication session. The session key $K_{S}$ is generated from random values exchanged between $L R$ and $L I$. With a shared session key $K_{S}, L R$ and $L I$ can securely exchange data, for instance using Advanced Encryption Standard (AES).

$$
\begin{aligned}
\text { LI } & : \text { Get current timestamp } T_{L I} \\
& : e_{I D}=H M A C_{H_{K}}\left(T_{L I}\right) \oplus I D_{L} \\
: & H_{T}=H M A C_{K_{L}}\left(T_{L I}\right) \\
: & \text { Calculate } H_{1_{i}}=H M A C_{I d_{T_{\text {Temp }}}}\left(T_{L I}\right) \forall I d_{T_{e m p}} \\
L R \stackrel{b_{1}}{\longleftarrow} L I: & H_{T}, e_{I D}, A R, W_{S}, T_{L I}
\end{aligned}
$$

$$
\begin{aligned}
L R: & \text { if } T_{Z}<T_{C} \text { or } T_{Z}<T_{L I} \text { or } T_{L I}<T_{C} \text { or } T_{0}<T_{L I} \\
& E N D S E S S I O N \\
: & H_{K}^{\prime}=H M A C_{K_{C}}\left(W_{S}\right) \\
: & I D_{L}^{\prime}=H M A C_{H_{K}^{\prime}}\left(T_{L I}\right) \oplus e_{I D} \\
: & K_{L}^{\prime}=H M A C_{K_{C}}\left(I D_{L}^{\prime}\|A R\| W_{S}\right) \\
: & H_{T}^{\prime}=H M A C_{K_{L}^{\prime}}\left(T_{L I}\right)
\end{aligned}
$$




$$
\begin{aligned}
& \text { if }\left(H_{T}^{\prime}==H_{T}\right) \quad \% \% L I^{\prime} \text { s Key } K_{L} \text { is valid } \\
& T_{C}=T_{L I} \\
& \text { else } \\
& \text { END SESSION } \\
& \text { Calculate } I d_{T e m p}=H M A C_{I d}\left(T_{0}\right) \\
& H_{1}=H M A C_{I d_{\text {Temp }}}\left(T_{L I}\right) \\
& \text { Generate random } R_{1} \\
& e_{1}=R_{1} \oplus I d_{T e m p} \\
& H_{2}=H M A C_{I d_{T e m p}}\left(R_{1} \| K_{L}^{\prime}\right) \\
& L I \stackrel{b_{2}}{\longleftarrow} L R \quad: \quad e_{1}, H_{1}, H_{2} \\
& L I \text { : Search for } H_{1} \text { in a list of } H_{1_{i}} \\
& R_{1}^{\prime}=e_{1} \oplus I d_{T e m p} \\
& H_{2}^{\prime}=H M A C_{I d_{\text {Temp }}}\left(R_{1}^{\prime} \| K_{L}\right) \\
& \text { if }\left(H_{2}^{\prime} \neq H_{2}\right) \\
& \text { END SESSION } \\
& \text { else } \% \% A R \text { is authentic } \\
& H_{R}=H M A C_{I d_{\text {Temp }}}\left(R_{1}^{\prime}\right) \\
& K_{S}=H M A C_{I d_{T e m p}}\left(R_{1}^{\prime} \| T_{L I}\right) \\
& L R \stackrel{b_{3}}{\longleftarrow} L I \quad: \quad H_{R} \\
& L R \quad: \quad H_{R}^{\prime}=H M A C_{I d_{\text {Temp }}}\left(R_{1}\right) \\
& \text { if }\left(H_{R}^{\prime}==H_{R}\right) \quad \% \% L I \text { is authentic } \\
& K_{S}=H M A C_{I d_{T e m p}}\left(R_{1} \| T_{L I}\right)
\end{aligned}
$$

Listing 2: Mutual authentication phase between LI and LR

\section{Protocol Validation}

We used Automated Validation of Internet Security Protocols and Applications (AVISPA) [4] tool to validate our protocol. AVISPA's current version integrates four back-ends, On-the-fly Model-Checker (OFMC), Constraint-Logic-based Attack Searcher (CL-AtSe), SAT-based ModelChecker (SATMC), and Tree Automata based on Automatic Approximations for the Analysis of Security Protocols (TA4SP). The attacker model is Dolev-Yao attacker [12] i.e an attacker has full capabilities over the newtork and can listen or intercept communication, inject new messages or modify messages in transit. The High Level Protocol Specification Language (HLPSL) [35] code for our protocol used in AVISPA is presented in Annex 1.

In Listing 3, AVISPA outputs SAFE from three back-ends, OFMC $C L-A t S e$, and SATMC. This implies that AVISPA could not reproduce any attack on our proposed protocol. AVISPA gives INCONCLUSIVE result in TA4SP back-end because TA4SP does not perform verification. Nevertheless, our protocol is still safe.

\begin{tabular}{ll}
\hline OFMC & : SAFE \\
CL-AtSe & $:$ SAFE \\
SATMC & $:$ SAFE \\
TA4SP & $:$ INCONCLUSIVE
\end{tabular}

Listing 3: Validation results after running our protocol in AVISPA

\section{Analysis of the Proposed Solution}

We analyze our proposed protocol in terms of performance, privacy and security.

\subsection{LR's Performance Analysis}

In our scenario, $L R$ is the most resource constrained device but holds all the sensitive information, hence we analyze its performance in the following areas:
Computational Cost: Our proposed protocol uses simple primitives such as comparison, XoR and HMAC. Among the chosen primitives, HMAC is more resource demanding. Moreover, HMAC demands more resources than a normal Hash function but guarantees optimal security by reducing the number of collisions compared to a normal Hash function [17].

Storage Cost: Storage is a critical issue in $L R$, hence our protocol requires only few space for storage as each device stores only minimal initial parameters $-K_{C}, I d$ and $T_{C}$, amounting to 288 bits ( 36 bytes) for $L R$. During runtime, our protocol requires a maximum total of 736 bits (92 bytes) of storage in $L R$ (corresponds to Line (18) of our protocol sequence).

Communication Cost: Constrained devices expend a lot of energy in transmitting and receiving information [22], [25], [28]. A protocol with fewer and shorter messages guarantees reduced energy consumption. Our protocol exchanges three messages during mutual authentication stage with a total of 992 bits (124 bytes).

\subsection{Security and Privacy Analysis}

We analyse our protocol against attack scenarios put forth in Section 4.2 This is a complementary analysis to AVISPA's validation as it analyses the mechanisms of the exchanged information in details. Game 1: $\beta$ masquerades as LI; Referring to Game 1 in Section 4.2 . $\beta$ 's objective is to send valid messages $b_{1}$ and $b_{3}$. That is, $\beta$ can either try to crack the key $K_{L}$ along with $I D_{L}$ or generate a valid message $b_{3}$ based on previously sniffed messages $b_{2}$ and $b_{3}$ during Phase 1.1.

For cracking key $K_{L}$ along with $I D_{L}, \beta$ is able to spoof $L I$. One of the solutions involves extracting values $H_{T}$ and $T_{L I}$ from a known message $b_{1}$ and try to crack $K_{L}$. This assumes that the HMAC function is not robust to collision attacks, which is contrary to our assumptions of Section 4.3 Alternativey, $\beta$ can combine messages $b_{1}, b_{2}$ and $b_{3}$ and try to deduce valuable information. However, all messages behave like random or pseudo-random strings. Indeed, $e_{1}$ is randomized thanks to random $R_{1}$. Values $e_{I D}, H_{T}, H_{2}$ and $b_{3}$ are HMAC outputs and, as stated in [14], they behave as pseudo-random strings and evolve independently from each other as their inputs are different. As such, whatever the number of sniffed messages $b_{1}, b_{2}$ and $b_{3}$, it is not possible to extract any kind of information, and the game can not succeed.

Game 2: $\beta$ tracks LR; Following Game 2 in Section 4.2. $L R_{i}$ responds for session $j$ with messages $e_{1 i j}$ and $e_{2 i j}$ which behave as random or pseudo-random strings. Such that, any response from $L R_{1}$ is semantically indistinguishable from responses of $L R_{2}$, and previous responses of $L R_{1}$. Hence, an adversary $\beta$ is unable to guess with a probability greater than 0.5 which $L R_{i}$ sent message $b 2$.

Game 3: $\beta$ depletes LR's resources; Game 3 in Section 4.2 is a form of Denial of Service (DoS) attack such that an adversary $\beta$ constantly queries $L R$ to utilize its resources and deplete its energy source. Our protocol tests message $b_{1}$ using four comparison operations, four HMAC operations, and one XoR operation to verify validity. Of these operations, HMAC consumes more energy as explained in section 6.1

Now, let us quantify the duration of time needed for $\beta$ to deplete an alkaine long-life $A A A$ battery with total energy of 5071 Joules [16]. If $L R$ conforms to IEEE 802.15.4 [6] with an antenna frequency of $2.4 \mathrm{GHz}$ band, data rate of $250 \mathrm{Kbps}$, and power consumption of $1.475 \mathrm{~W}$ in receive mode [9], then it will take approximately $2 \mathrm{~ms}$ (milliseconds) to receive 480 bits of data sent in message $b_{1}$ by dissipating $1.475^{*} 0.002=0.003$ Joules. According to [27], HMAC function consumes $1.16 \mu \mathrm{J}$ (microjoules) per byte of data. Parameters used in calculations have a total of 960 bits or 120 bytes for $H_{K}^{\prime}$ (Equation (16)), and $I D_{L}^{\prime}$ (Equation (17)), $K_{L}^{\prime}$ (Equation (18)), and $H_{T}^{\prime}$ (Equation (19)). As such, each request from $\beta$ costs $1.16^{*} 120=139.2 \mu \mathrm{J}$, which makes a total of 0.003 Joules for receiving and calculations. With this consumption rate, it will take 1,706,718 rounds to deplete the battery. Suppose $\beta$ sends message $b_{1}$ to $L R$ every 1 second, it will take around 20 days to deplete the battery, with most of the energy being spent in receiving message $b_{1}$. Hence this game cannot succeed. 


\section{Conclusion}

In this article we presented serverless lightweight mutual authentication protocol for small mobile resource computing devices. The protocol has been thoroughly explained, analyzed and its advantages outlined.

The originality of the protocol is based on the idea that a resource constrained device can mutually authenticate with another device without sharing any information. Our lightweight protocol uses simple primitives such as XoR, comparison and HMAC function, during mutual authentication and it also requires few storage space in each communicating party.

Thorough analysis was done to theoretically verify the security and performance properties of the protocol. The security properties have also been formally validated using AVISPA tool.

\section{References}

1. Sarah Abughazalah et al. A mutual authentication protocol for low-cost rfid tags formally verified using casperfdr and avispa. In Information Science and Technology (ICIST), 2013 International Conference on, pages 44-51. IEEE, 2013

2. Sheikh Ahamed et al. Erap: Ecc based rfid authentication protocol. In Future Trends of Distributed Computing Systems, 2008. FTDCS'08. 12th IEEE International Workshop on, pages 219-225. IEEE, 2008

3. Moshaddique Al Ameen et al. Security and privacy issues in wireless sensor networks for healthcare applications. Journal of medical systems, 36(1):93-101, 2012.

4. Alessandro Armando et al. The avispa tool for the automated validation of internet security protocols and applications. In Computer Aided Verification, pages 281-285. Springer, 2005.

5. Luigi Atzori, Antonio Iera, and Giacomo Morabito. The internet of things: A survey. Computer networks, 54(15):2787-2805, 2010.

6. Bruno Bougard et al. Energy efficiency of the ieee 802.15. 4 standard in dense wireless microsensor networks: Modeling and improvement perspectives. In Design, Automation, and Test in Europe, pages 221-234. Springer, 2008

7. David Carman et al. Constraints and approaches for distributed sensor network security (final). DARPA Project report,(Cryptographic Technologies Group, Trusted Information System, NAI Labs), 1:1 2000

8. Christy Chatmon et al. Secure anonymous rfid authentication protocols. Florida State University Department of Computer Science, Tech. Rep, 2006.

9. Jyh-Cheng Chen et al. A comparison of mac protocols for wireless local networks based on battery power consumption. In INFOCOM'98. Seventeenth Annual Joint Conference of the IEEE Computer and Communications Societies. Proceedings. IEEE, volume 1, pages 150-157. IEEE, 1998.

10. Tien-Ho Chen et al. A robust mutual authentication protocol for wireless sensor networks. Etri Journal, 32(5):704-712, 2010.

11. Miaolei Deng et al. Weakness in a serverless authentication protocol for radio frequency identification. In Mechatronics and Automatic Control Systems, pages 1055-1061. Springer, 2014.

12. Danny Dolev et al. On the security of public key protocols. Information Theory, IEEE Transactions on, 29(2):198-208, 1983.

13. Donald Eastlake and Paul Jones. Us secure hash algorithm 1 (sha1), 2001

14. Pierre-Alain Fouque et al. Hmac is a randomness extractor and applications to tls. In Proceedings of the 2008 ACM symposium on Information, computer and communications security, pages 21-32. ACM, 2008.

15. Md Endadul Hoque et al. Enhancing privacy and security of rfid system with serverless authentication and search protocols in pervasive environments. Wireless personal communications, 55(1):65-79, 2010

16. http://www.allaboutbatteries.com/Energy tables.html. Battery types, energy and consumptions, June 2011

17. Marcio Juliato et al. An efficient fault-tolerance technique for the keyed-hash message authentication code. In Aerospace Conference, 2010 IEEE, pages 1-17. IEEE, 2010.

18. Hermann Kopetz. Internet of things. In Real-time systems, pages 307-323. Springer, 2011.

19. Chin-Feng Lee et al. Server-less rfid authentication and searching protocol with enhanced security. International Journal of Communication Systems, 25(3):376-385, 2012.

20. Mingyan Li et al. Multi-domain rfid access control using asymmetric key based tag-reader mutual authentication. In ICAS2008-Proceedings of the 26th international Congress of the Aeronautical Sciences, 2008

21. Luon-Chang Lin et al. Lightweight and serverless rid authentication and search protocol. In 2009 Second International Conference on Computer and Electrical Engineering, volume 2, pages 95-99, 2009.

22. Gang Lu et al. Performance evaluation of the ieee 802.15. 4 mac for low-rate low-power wireless networks. In Performance, Computing, and Communications, 2004 IEEE International Conference on pages 701-706. IEEE, 2004

23. Parikshit Mahalle et al. Identity establishment and capability based access control (iecac) scheme for internet of things. In Wireless Personal Multimedia Communications (WPMC), 2012 15th International Symposium on, pages 187-191. IEEE, 2012.
24. Bruce Ndibanje et al. Security analysis and improvements of authentication and access control in the internet of things. Sensors, 14(8):14786-14805, 2014.

25. Trevor Pering et al. Coolspots: reducing the power consumption of wireless mobile devices with multiple radio interfaces. In Proceedings of the 4th international conference on Mobile systems, applications and services, pages 220-232. ACM, 2006.

26. Selwyn Piramuthu. Rfid mutual authentication protocols. Decision Support Systems, 50(2):387-393 2011

27. Nachiketh R Potlapally et al. Analyzing the energy consumption of security protocols. In Proceedings of the 2003 international symposium on Low power electronics and design, pages 30-35. ACM 2003.

28. Gregory Pottie et al. Wireless integrated network sensors. Communications of the ACM, 43(5):51-58, 2000.

29. Mohsen Pourpouneh et al. An improvement over a server-less rfid authentication protocol. Inter national Journal of Computer Network and Information Security (IJCNIS), 7(1):31, 2014.

30. Masoumeh Safkhani et al. On the security of tan et al. serverless rfid authentication and search protocols. In Radio Frequency Identification. Security and Privacy Issues, pages 1-19. Springer, 2013.

31. Chiu C Tan et al. Body sensor network security: an identity-based cryptography approach. In Proceedings of the first ACM conference on Wireless network security, pages 148-153. ACM, 2008.

32. Chiu Chiang Tan et al. Severless search and authentication protocols for rfid. In Pervasive Computing and Communications, 2007. PerCom'07. Fifth Annual IEEE International Conference on, pages 3-12. IEEE, 2007.

33. Caimu Tang et al. An efficient mobile authentication scheme for wireless networks. Wireless Communications, IEEE Transactions on, 7(4):1408-1416, 2008.

34. Gene Tsudik. Ya-trap: Yet another trivial rfid authentication protocol. In Pervasive Computing and Communications Workshops, 2006. PerCom Workshops 2006. Fourth Annual IEEE International Conference on, pages 4-pp. IEEE, 2006.

35. David von Oheimb. The high-level protocol specification language hlpsl developed in the eu project avispa. In Proceedings of APPSEM 2005 Workshop, 2005.

36. John Walters et al. Wireless sensor network security: A survey. Security in distributed, grid, mobile, and pervasive computing, 1:367, 2007

\section{Annex 1: HLPSL code for Mutual Authentication Protocol}

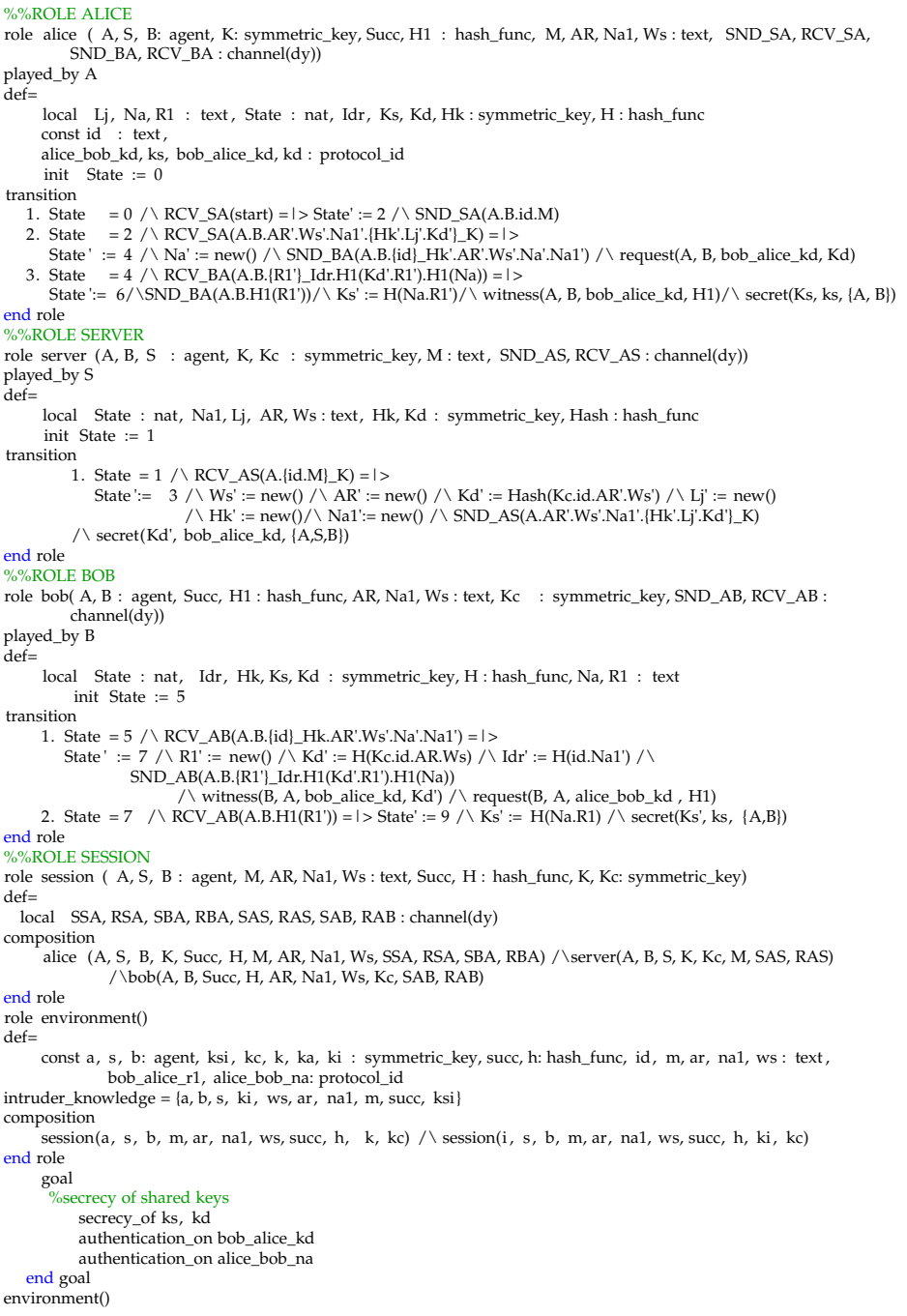

